Radiologe 2011 · 51:1015

DOI 10.1007/s00117-011-2245-4

Online publiziert: 22. Oktober 2011

(c) Springer-Verlag 2011

Redaktion

S. Delorme

\section{Originalpublikation}

Nasis A et al (2011) Acute chest pain investigation: utility of cardiac CT angiography in guiding troponin measurement. Radiology 260:381-389

Die CT des Herzens ist eine relativ neue Methode, die in den letzten 10 Jahren eine rasante Entwicklung genommen hat. $\mathrm{Zu}$ den klinischen Einsatzmöglichkeiten zählen heute in erster Linie das Kalziumscoring und die nichtinvasive CT-Angiographie der Koronararterien (CTCA). Troponin I ist ein Proteinkomplex, der in der Herzmuskelzelle vorkommt. Werden die Kardiomyozyten z. B. durch einen Herzinfarkt geschädigt, wird das kardiale Troponin in den Blutkreislauf freigesetzt. Der Nachweis eines erhöhten Troponinspiegels im Blut ist ein sensitiver Marker für einen Herzmuskelschaden.

Nasis et al. entwickelten einen neuen diagnostischen Algorithmus zur Untersuchung von Patienten mit Angina pectoris und geringer bis mittlerer Wahrscheinlichkeit einer koronaren Herzkrankheit (KHK). Der Grundgedanke basiert auf der Kombination der oben genannten Untersuchungen. Nach einer allgemeinen körperlichen Untersuchung, einem Elektrokardiogramm (EKG) und einer initial negativen Troponinbestimmung erfolgte eine CTCA. Dabei wurde in der prospektiven Studie eine obstruktive KHK als eine Reduktion des Koronararteriendurchmessers $\geq 50 \%$ definiert.

Ausschlusskriterien waren ein akutes Koronarsyndrom (koronare Herzkrankheit, Lungenödem, ventrikuläre Tachykardie), ein STEMI („ST-elevation myocardial infarction") im EKG und allgemeine

\author{
M. Karul · J. Yamamura \\ Klinik und Poliklinik für Diagnostische und Interventionelle Radiologie, \\ Universitätsklinikum Hamburg-Eppendorf, Hamburg
}

\title{
Neues diagnostisches Konzept bei Patienten mit Angina pectoris
}

Kontraindikationen für eine CTCA (z. B. allergische Reaktion auf jodhaltige Kontrastmittel oder Schwangerschaft).

Bei den 203 Studienpatienten wurden in der CTCA folgende Resultate ermittelt:

- 65 Patienten waren unauffällig und konnten bereits nach einmaliger Troponinbestimmung entlassen werden.

- 107 Patienten wiesen keine signifikante Reduktion des Koronararteriendurchmessers auf und konnten nach wiederholten Troponinbestimmungen entlassen werden.

- 31 Patienten hatten entweder moderate $(50-70 \%)$ oder schwere $(>70 \%)$ Reduktionen des Koronararteriendurchmessers. Diese Patienten wurden zur Diagnostik der morphologischen Verhältnisse der Koronararterien und zur Lokalisierung von Stenosen einer invasiven Koronarangiographie zugeführt. In dieser weiterführenden Untersuchung bestätigte sich bei 27 Patienten ein richtig-positiver und bei den restlichen 4 Patienten ein falsch-positiver Befund.

Aus den Ergebnissen schlussfolgern die Autoren, dass in Zusammenschau einer unauffälligen CTCA mit einer wiederholt negativen Troponinbestimmung ein akuter Herzinfarkt sicher ausgeschlossen werden kann. In der Folge sei die Krankenhausaufenthaltsdauer einschließlich der daraus resultierenden Kosten deutlich reduziert.

Insgesamt müssen das Studiendesign und die Bewertung der Ergebnisse kritisch gesehen werden. Wenn die Patienten ohnehin kein hohes Risikoprofil (Diabetes, Hyperlipidämie, Hypertension, $\mathrm{Ni}$ kotinabusus, familiäre Häufung) für eine KHK aufweisen, muss die rechtfertigende Indikation zur CTCA kritisch hinterfragt werden. Des Weiteren wurde die Kardio-MRT völlig außer Acht gelassen. Diese Untersuchung ermöglicht Aussagen zur Schlagfunktion und der Kontraktionsfähigkeit des Herzens. Nach i.-v.Kontrastmittelgabe (Gd-DTPA) kann die Kardio-MRT zur Beurteilung der Vitalität und zum Nachweis bzw. Ausschluss von Durchblutungsstörungen des Myokards eingesetzt werden. Zudem können in der Kardio-MRT weitere Fragestellungen beispielsweise nach einer dilatativen Kardiomyopathie, einer Myokarditis oder nach Vitien beantwortet werden.

Zusammenfassend ist an dem diagnostischen Konzept positiv hervorzuheben, dass $85 \%$ der Patienten mit einem zuverlässigen Ausschluss eines Herzinfarkts die Notaufnahme nach kurzer Zeit (durchschnittlich 6-9 h) wieder verlassen konnten. Da die hämodynamische Relevanz der Stenosen nur eingeschränkt beurteilbar ist, könnte eine nachgeschaltete Kardio-MRT bei den übrigen $15 \%$ eine Befunderweiterung bringen.

\section{Korrespondenzadresse \\ M. Karul}

Klinik und Poliklinik für Diagnostische und Interventionelle Radiologie,

Universitätsklinikum Hamburg-Eppendorf,

Martinistr. 52, 20246 Hamburg

mkarul@uke.uni-hamburg.de

Interessenkonflikt. Der korrespondierende Autor gibt an, dass kein Interessenkonflikt besteht. 